\title{
Is SDSS J094533.99+100950.1 higher mass equivalent to narrow-line Seyfert 1 galaxies?
}

\author{
Krzysztof Hryniewicz \\ Nicolaus Copernicus Astronomical Center, Warsaw (Poland) \\ E-mail: krhrecamk.edu.pl
}

\begin{abstract}
SDSS J094533.99+100950.1 is an example of a weak line quasar (WLQ). Two hypotheses explaining the WLQ phenomena are present in the literature: high accretion rate (which is the case of PHL 1811) or not fully developed broad emission line region (BLR) (which could be due to the evolutionary state - reactivation phase). What PHL 1811 and WLQ have in common are very low equivalent widths of the high-ionization emission lines like CIV or HeII, typical iron emission, radio quiescence and X-ray weakness. The explanation of the atypical quasar properties could be a super-Eddington accretion rate. In addition, the ionizing radiation source can be obscured by a wind or other medium on its way to the BLR. Another possibility is that due to the evolution of the active phase in AGN the emitting matter can have unusual properties. The BLR could not be fully developed in the state before the wind is launched, thus occupying a compact region. To resolve the puzzle, spectral analysis, photoionization simulations, accretion disk continuum fitting and black hole spin estimation have been done.
\end{abstract}

Narrow-Line Seyfert 1 Galaxies and their place in the Universe, April 04-06, 2011

Milan, Italy

\footnotetext{
* Speaker.
} 


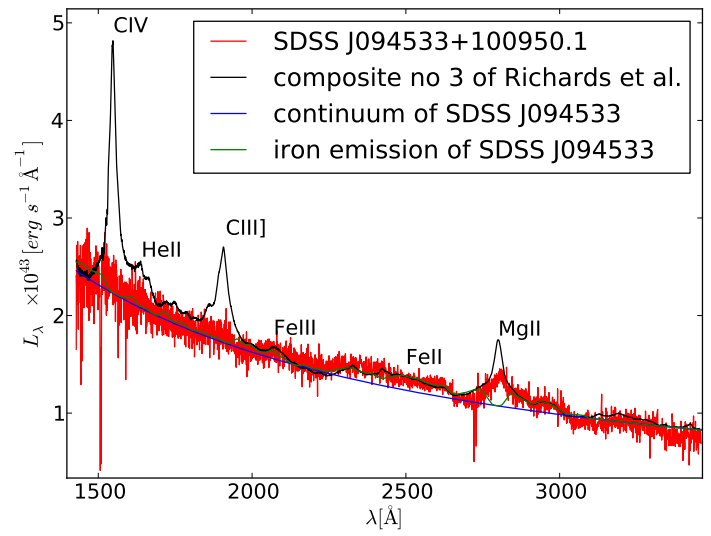

Figure 1: The restframe spectrum of SDSS J094533.99+100950.1 corrected for Galactic reddening (red line), its fitted, underlying continuum (blue line) and iron emission (green line). For comparison, in black, the composite spectrum (no. 3) [9] is shown, scaled to match the SDSS J094533.99+100950.1 spectrum.

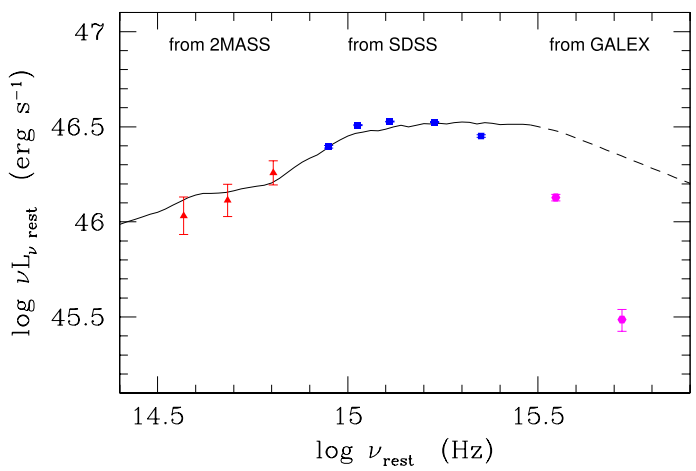

Figure 2: The broad band SED of SDSS J094533.99+100950.1. The photometric IR data points (triangles) come from 2MASS $\left(\mathrm{J}, \mathrm{H}, \mathrm{K}_{\mathrm{s}}\right.$ colours). Optical photometry (squares) is from SDSS (u,g,r,i,z filters). The NUV and FUV points (hexagons) are from GALEX. For comparison the line shows the SED from [3].

\section{Introduction}

SDSS J094533.99+100950.1 is an exceptional quasar. It has extremely weak medium-ionization (CIII], AlIII) and high-ionization lines (CIV, HeII) but typical FeII emission and near-UV powerlaw continuum [5]. In Figure 1 we present its spectrum compared to a quasar composite from [9]. Although MgII line seems to be weaker than in mean quasar spectra, its width is comparable to mean quasar line. This allows virial black hole mass estimation. Latest estimation of the black hole mass based on MgII line width and continuum luminosity is $M=2.9_{-1.9}^{+5.5} \times 10^{9} M_{\odot}$ (based on [11] formulas) thus $\dot{m}=0.17$. 


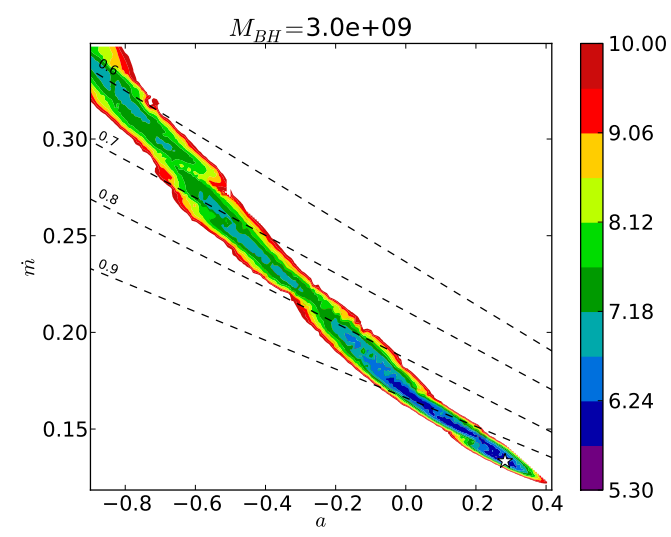

Figure 3: The contour errors for the Kerr parameter, $a$, the accretion rate, $\dot{m}$, and the black hole mass, $M=3 \times 10^{9} M_{\odot}$. The star indicates the position of the best fit solution, and dashed lines show the best fit values of the cosine of the disk inclination.

\section{Fitting an accretion disk continuum}

The opt-UV continuum is soft and accretion-disk-like. We plot photometric points together with the QSO SED in Figure 2. Authors in the [2] have fitted an accretion disk continuum around a Kerr black hole and put constraints on its parameters. For $M=2.7 \times 10^{9} M_{\odot}$ they obtain moderate spin value, $a=0.46$, accretion rate consistent with estimation from MgII line, $\dot{m}=0.135$, and low inclination, $\cos i=0.972$. Figure 3 shows an example of the $\chi^{2}$ color-map, dashed lines indicates $\cos i$.

\section{Photoionization simulations}

To perform photoionization-based BLR parameters estimation we used Cloudy code [4]. As an initial input we used observed continuum parametrized as:

$$
f_{v}=A v^{\alpha_{\mathrm{Uv}}} \exp \left(-\frac{h v}{k T_{\mathrm{BB}}}\right) \exp \left(-\frac{h v}{k T_{\mathrm{IR}}}\right)+B v^{\alpha_{\mathrm{x}}}
$$

with the following AGN parameters: $T_{\mathrm{BB}}=40000 \mathrm{~K}, \alpha_{\mathrm{UV}}=0.77$, and assumed X-ray spectral part described by parameters: $\alpha_{\mathrm{ox}}=-1.7$, and $\alpha_{\mathrm{x}}=-1$. In the initial setup we assumed solar abundance.

\section{BLR as a single cloud}

As we see in Figure 4 all line luminosity ratios meet in a compact region (intersections of solid lines at $\log n_{H}\left[\mathrm{~cm}^{-3}\right] \approx 11, \log r[\mathrm{~cm}] \approx 18$ ). Absolute line luminosities agree quite well except one line (HeII could be underestimated), however MgII line could be overestimated (due to blended FeII emission under MgII). Intersection position almost does not depends on the hydrogen column density [6]. 


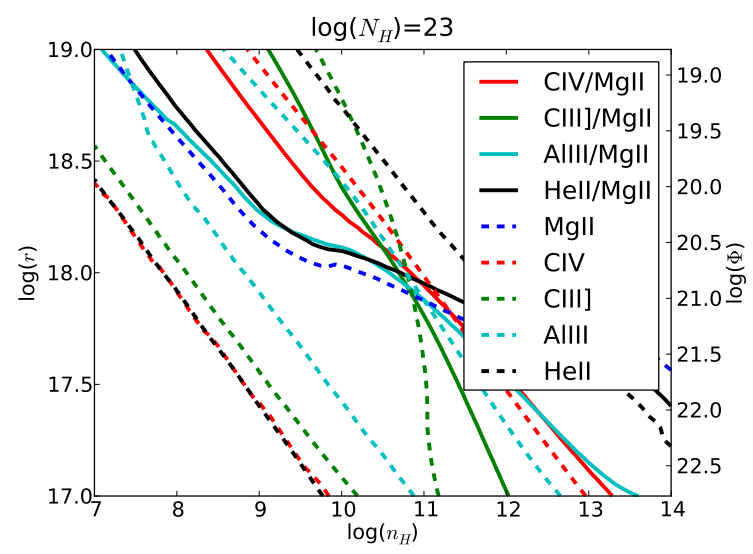

Figure 4: The observed relative and absolute lines luminosities as reproduced in simulations in the radius local density plane.

On the other hand derived distance between BH and BLR based on the reverberation mapping formula by [7]:

$$
\log \frac{R_{\mathrm{BLR}}}{\text { lt.days }}=(1.27 \pm 0.10)+(0.58 \pm 0.10) \log \frac{L_{3000 \mathrm{~A}}}{10^{44} \mathrm{erg} \mathrm{s}^{-1}}
$$

gives $\log R_{\mathrm{BLR}}[\mathrm{cm}]=17.98$ and this value agrees well with the preferred location in our single cloud model.

\section{Comparison to disk vertical structure}

Simulation of the disk vertical structure was performed based on the code described by [10]. Parameters of the accretion disk atmosphere agrees very well with those preferred by line luminosities $-\log n_{H}\left[\mathrm{~cm}^{-3}\right] \approx 11, \log r[\mathrm{~cm}] \approx 18$. This supports hypothesis in which BLR is compact and not fully developed but rather appears as an "expanding" accretion disk atmosphere.

\section{Locally Optimally Emitting Clouds}

Assuming BLR as a distribution of clouds over a whole parametric space, line luminosity is computed as integral of a line flux $F$ :

$$
L_{\text {line }} \propto \iint r^{2} F(r, n) r^{\gamma} n^{\beta} \mathrm{d} n \mathrm{~d} r .
$$

The $\gamma$ and $\beta$ for which observed luminosities are reproduced by simulations are shown in Figure 6 It is relatively easy to match all observed line luminosities however it demands $\gamma$ and $\beta$ values to be more extreme than those adopted by [1] and other authors. 


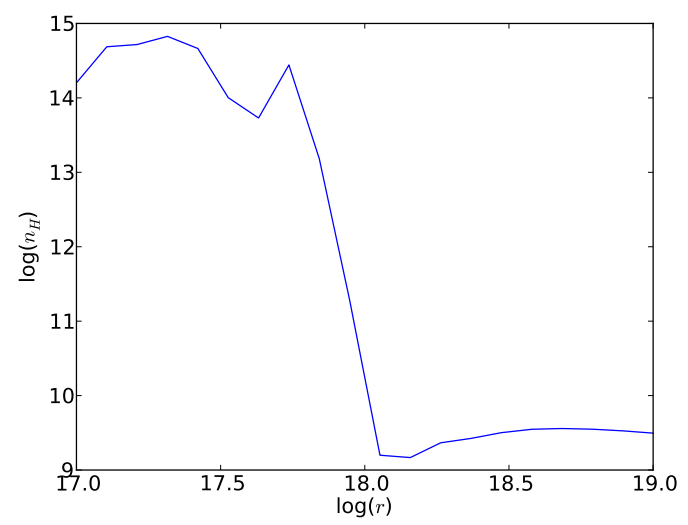

Figure 5: The local hydrogen density distribution along radius, in the disk atmosphere at $\tau=2 / 3$, computed in the accretion disk vertical structure simulations.

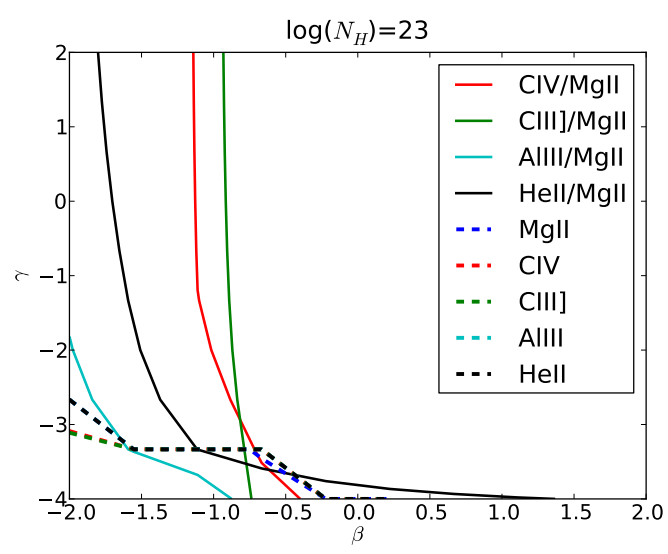

Figure 6: The lines show dependence of the integrated lines luminosities on clouds distribution indexes $\beta$ and $\gamma$.

\section{Summary}

The black hole and accretion disk parameters of SDSS J094533.99+100950.1 are within ranges typical among quasars. Preliminary results of photoionization simulations show that its weak emission lines can be explained by a compact BLR in early developing phase scenario. Agreement between prefered position and local density in the single cloud model with values derived from the reverberation mapping formula and the disk vertical structure simulations may support hypothesis of early evolutionary stage in AGN activation process. Another possibility is that in SDSS J0945+1009 there is more extreme distribution of the BLR clouds than in case of normal quasars. It is necessary to further investigate how sensitive the result is on the simulation setup and also on potential inaccuracies in the line fitting (due to blending $\mathrm{MgII}$ with $\mathrm{Fe}$ ). The high accretion rate scenario, valid for PHL 1811 NLS1 and well explaining its weak lines [8], seems not be the case of SDSS J0945+1009, as derived accretion rate is rather low. 


\section{Acknowledgments}

This work was supported in part by grant N N203 380136. I would like to thank Bożena Czerny for valuable discussions and her advices.

\section{References}

[1] J. Baldwin, G. Ferland, K. Korista, D. Verner: Locally Optimally Emitting Clouds and the Origin of Quasar Emission Lines, ApJ, 455L, 119 (1995)

[2] B. Czerny, K. Hryniewicz, M. Nikołajuk, A. Sądowski, 2011, Constraints on the black hole spin in the quasar SDSS J094533.99+100950.1, MNRAS accepted

[3] M. Elvis et al., 1994, Atlas of quasar energy distributions, ApJS, 95, 1

[4] G. J. Ferland, K. T. Korista, D. A. Verner, J. W. Ferguson, J. B. Kingdon, E. M. Verner, 1998, CLOUDY 90: Numerical Simulation of Plasmas and Their Spectra, PASP, 110, 761

[5] K. Hryniewicz, B. Czerny, M. Nikołajuk, J. Kuraszkiewicz, 2010, SDSS J094533.99+100950.1 - the remarkable weak emission line quasar, MNRAS, 404, 2028

[6] K. Hryniewicz, M. Nikołajuk, G. J. Ferland, Constraints on the properties of the Broad Line Region in the Weak Line Quasar SDSS J094533.99+100950.1, in preparation

[7] M.-Z. Kong, X.-B. Wu, R. Wang, J.-L. Han, 2006, Estimating Black Hole Masses of AGNs using Ultraviolet Emission Line Properties, Chin. J. Astron. Astrophys, 6, 396

[8] K. M. Leighly, J. P. Halpern, E. B. Jenkins, D. Casebeer, 2007, The Intrinsically X-Ray-weak Quasar PHL 1811. II. Optical and UV Spectra and Analysis, ApJS, 173, 1

[9] G.T. Richards et al., 2003, Red and Reddened Quasars in the Sloan Digital Sky Survey, AJ, 126, 1131

[10] A. Różańska, B. Czerny, P. T. Życki, G. Pojmański, 1999, Vertical structure of accretion discs with hot coronae in active galactic nuclei, MNRAS, 305, 481

[11] J.-G. Wang, X.-B. Dong, T.-G. Wang, L. Ho, W. Yuan, H. Wang, K. Zhang, S. Zhang, H. Zhou, 2009, Estimating Black Hole Masses in Active Galactic Nuclei Using the Mg II $\lambda 2800$ Emission Line, ApJ, 707,1334 